\title{
Tricuspid valve repair in hypoplastic left heart syndrome
}

Richard G. Ohye, $\mathrm{MD}^{\mathrm{a}}$

Carlen A. Gomez, MD ${ }^{b}$

Caren S. Goldberg, MD, MS ${ }^{\text {b }}$

Holly L. Graves, BA ${ }^{\text {a }}$

Eric J. Devaney, MDa

Edward L. Bove, MD ${ }^{\mathrm{a}}$

From the Divisions of Pediatric Cardiovascular Surgery ${ }^{\mathrm{a}}$ and Pediatric Cardiology, ${ }^{\mathrm{b}}$ University of Michigan Medical School, Ann Arbor, Mich.

Read at the Eighty-third Annual Meeting of The American Association for Thoracic Surgery, Boston, Mass, May 4-7, 2003.

Received for publication April 30, 2003; revisions requested July 7, 2003; accepted for publication July 22, 2003.

Address for reprints: Richard G. Ohye, MD, F7830 Mott/0223, 1500 East Medical Center Dr, Ann Arbor, MI 48109 (E-mail: ohye@umich.edu).

J Thorac Cardiovasc Surg 2004;127:465-72 $0022-5223 / \$ 30.00$

Copyright $(\odot) 2004$ by The American Association for Thoracic Surgery

doi:10.1016/j.jtcvs.2003.07.053

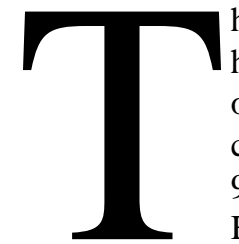

Objectives: Currently, the survival for the Norwood procedure for hypoplastic left heart syndrome is approximately $90 \%$ in selected centers. However, the development of tricuspid regurgitation remains a significant obstacle to successful staged repair in a subset of these patients. The results of tricuspid valve repair in this challenging patient population remain largely unknown.

Methods: Twenty-eight patients with significant (3-4+) tricuspid regurgitation after the Norwood procedure required tricuspid valve repair from August 1995 through December 2002. The clinical and Doppler-echocardiographic data were reviewed to determine the efficacy of repair and patient outcome.

Results: Follow-up was $96 \%$ complete (27/28). Patients were divided into 2 groups on the basis of tricuspid regurgitation at late follow-up: those with a successful late outcome $(0-2+)$ and those with a poor outcome $(3-4+)$. There were $17(63 \%)$ patients with a successful result and 10 (37\%) with an adverse outcome. Age, weight, follow-up duration, valve anatomy, and stage of palliation were not significantly different between groups. Early postoperative 0 to $2+$ regurgitation was associated with a durable result $(P=.012)$ and preserved ventricular function $(P=$ $.04)$. Need for repair other than a partial annuloplasty was predictive of a poor outcome $(P=.04)$. Overall survival was $67 \%(18 / 27)$. Survival was $94 \%(16 / 17)$ for patients with a successful late result versus $20 \%(2 / 10)$ for those with a poor outcome $(P=.0002)$.

Conclusions: Tricuspid valve repair can be accomplished in this challenging patient population with excellent results. Successful tricuspid valve repair is predictive of continued good valve function and preserved right ventricular function. Successful valve repair at late follow-up predicts excellent late survival.

icant, with mortalities of $4 \%$ to $15 \%$ Five-year survivals for HLHS, even at centers reporting the best Norwood procedure outcomes, remain approximately $75 \% .{ }^{1,5}$ In addition to the deaths, some patients are unable to progress through the 3 stages of reconstruction and might require cardiac transplantation or have no options for further therapy. There are many causes for these mortalities and morbidities after the Norwood procedure. These factors include increased pulmonary vascular resistance, cardiac arrhythmia, coronary artery insufficiency, right ventricular (RV) failure, RV volume overload caused by shunt-dependent physiology, and tricuspid valve regurgitation (TR). Many of these 


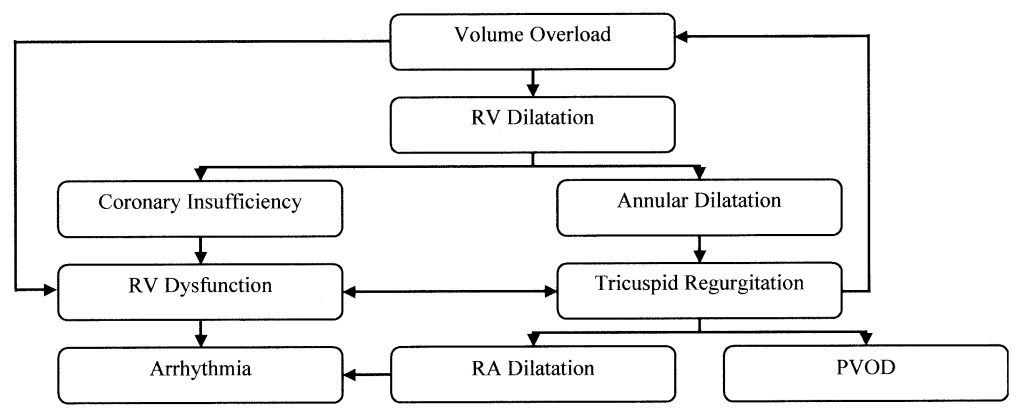

Figure 1. The relationships between factors negatively affecting mortality and morbidity in HLHS. PVOD, Pulmonary vascular obstructive disease.

factors are interrelated and might form feedback loops, which serve to propagate their adverse effects on patients with HLHS (Figure 1).

TR is of particular interest because it is one potential area in which surgical intervention might improve outcomes. TR might be due to an intrinsic abnormality of valve anatomy, or it might be due to any of the factors outlined above, such as RV dysfunction or dilatation. In addition, the morphologic tricuspid valve is subjected to systemic pressure rather than pulmonary workloads. Because of the multifactorial nature of the cause of TR, simple repair of the valve might not address the underlying problem and hence fail to improve the TR or patient outcome. To address this issue, we performed a retrospective review of all patients undergoing a tricuspid valve repair to assess early and late outcome, as well as procedural and anatomic variables predictive of successful repair.

\section{Methods}

\section{Study Design}

A retrospective analysis was performed on all patients with HLHS undergoing a tricuspid valve repair after a Norwood operation to determine the results of tricuspid valve repair in HLHS. The study hypothesis was that tricuspid valve repair is an effective means of treating TR in patients with HLHS. The primary end point was the degree of TR present at last follow-up, irrespective of nonvalverelated death or transplantation. Tricuspid regurgitation was characterized on a 0 to $4+$ grading scale, which is similar to that commonly used for mitral regurgitation. The grading scale was defined as follows: 0 , none; $1+$, mild (narrow regurgitant jet at the orifice [ $<2 \mathrm{~mm}$ ], single jet); $2+$, mild to moderate (wider jet area at orifice $[<4 \mathrm{~mm}]$, multiple jets, or both, mild atrial enlargement); $3+$, moderate to severe (wide jet orifice, jet reaches back wall of atrium, moderate atrial enlargement); and $4+$, severe (wide jet orifice, jet reaches back wall of atrium, reversal of flow in the pulmonary or hepatic veins, severe atrial enlargement). A successful outcome was defined as 0 to $2+$ TR and an adverse outcome as 3 to $4+$ TR or need for valve replacement. RV function was graded on a subjective scale as normal, mildly decreased, moderately decreased, and severely decreased. The valve anatomy responsible for the TR, such as leaflet prolapse or leaflet tethering, was noted. The diameter of the tricuspid valve, expressed as a $z$ value, was also recorded for all patients by using standard calculations to assess the degree of annular dilatation. ${ }^{6}$ These variables (degree of TR, RV function, valve anatomy, and tricuspid valve $z$ value) were assessed preoperatively, at discharge after repair, and at latest follow-up. A single echocardiographer reviewed all echocardiograms.

Secondary aims involved comparing the groups with a successful and unsuccessful result as defined above. Variables for comparison included patient demographics, valve anatomy, operative technique, RV function, and immediate postoperative result. The other secondary aims were to evaluate the durability of successful repair and survival.

\section{Patients}

From August 1994 through December 2002, 475 patients with HLHS underwent operations at the C. S. Mott Children's Hospital of the University of Michigan Health System. Of these operations, 28 had undergone a tricuspid valve repair for greater than $2+$ TR after a Norwood procedure. In general, valve repair was undertaken for the presence of 3 to $4+\mathrm{TR}$, regardless of symptoms, to optimize the patient's hemodynamics and preserve RV function. Only patients with classic HLHS, as defined by right ventricledependent systemic circulation and atresia or hypoplasia of the aortic valve, were enrolled. Patient demographics recorded included age at repair, weight at repair, stage of palliation, and length of follow-up. Surgical variables include type of repair, concomitant procedures, and operative morbidity or mortality.

\section{Surgical Techniques}

Surgical techniques were individualized to the patient's valve pathology on the basis of the opinion of the operating surgeon. Standard methods of cardiopulmonary bypass were used, with initial examination of the tricuspid valve performed by filling the right ventricle with saline. Intraoperative determination was made of the location or locations of regurgitation, the diameter of the annulus, leaflet prolapse, and leaflet tethering. Coupled with the preoperative echocardiogram, intraoperative echocardiogram, or both, appropriate methods of repair were then selected. Partial annuloplasty was commonly applied for patients with anterior leaflet prolapse or annular dilatation with failure of leaflet coaptation and generalized central regurgitation. This technique in- 

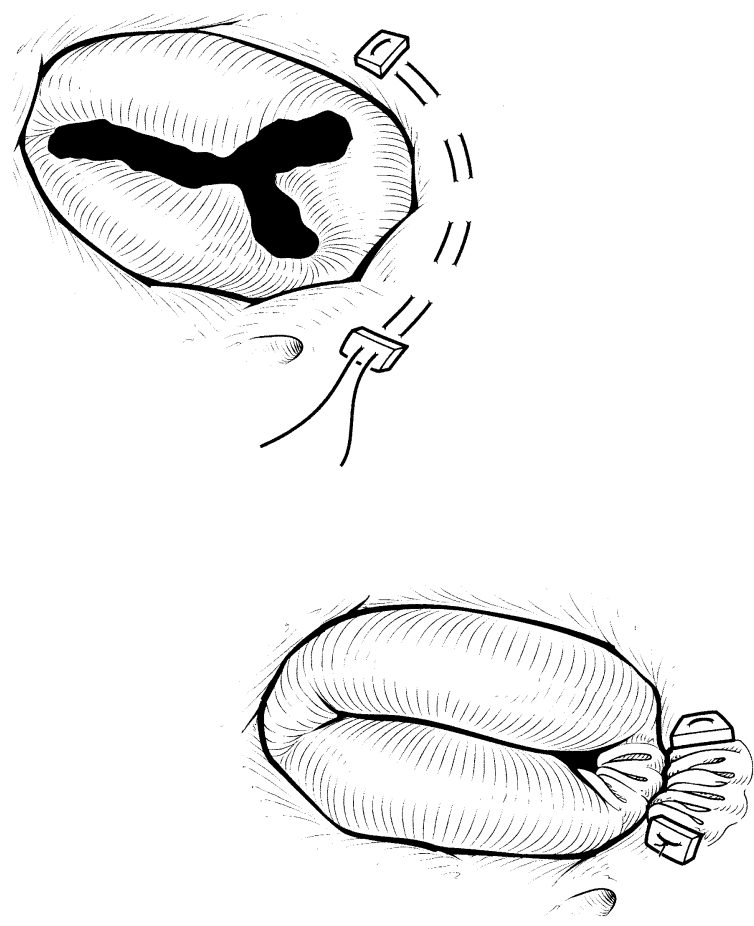

Figure 2. Partial annuloplasty.

volves running parallel mattress sutures along the annulus from the anteroposterior commissure to the posteroseptal commissure (Figure 2). The annuloplasty essentially obliterates the posterior leaflet, forming a bicuspid valve. Localized areas of leaflet prolapse were repaired by using partial commissure closure, and individual clefts or defects in the leaflets were closed primarily (Figure 3). Ring annuloplasty was also used, with a flexible partial ring. Less frequently used techniques included chordal shortening $(n=3)$, papillary muscle advancement $(\mathrm{n}=1)$, and mobilization of the septal leaflet $(n=1)$. The techniques were not mutually exclusive, and any individual patient might have undergone more than one repair method.

\section{Statistical Analysis}

Differences in dichotomous variables were compared by using the Fisher exact test. Wilcoxon rank sum tests were performed to compare the differences in ordinal variables, such as TR and RV dysfunction. TR in the postoperative period was dichotomized to $2+$ TR or less and greater than 2+ TR. The sensitivity, specificity, positive predictive value, and negative predictive value were then calculated for the finding of postoperative TR of greater than $2+$.

\section{Results}

\section{Patients}

Of the 28 patients who underwent a tricuspid valve repair for significant TR after a Norwood procedure, 27 (96\%) were available for follow-up. Median follow-up for the entire group was 20 months (range, 1-82 months). The patients were subsequently divided into groups on the basis of whether they had a successful late outcome, as defined by

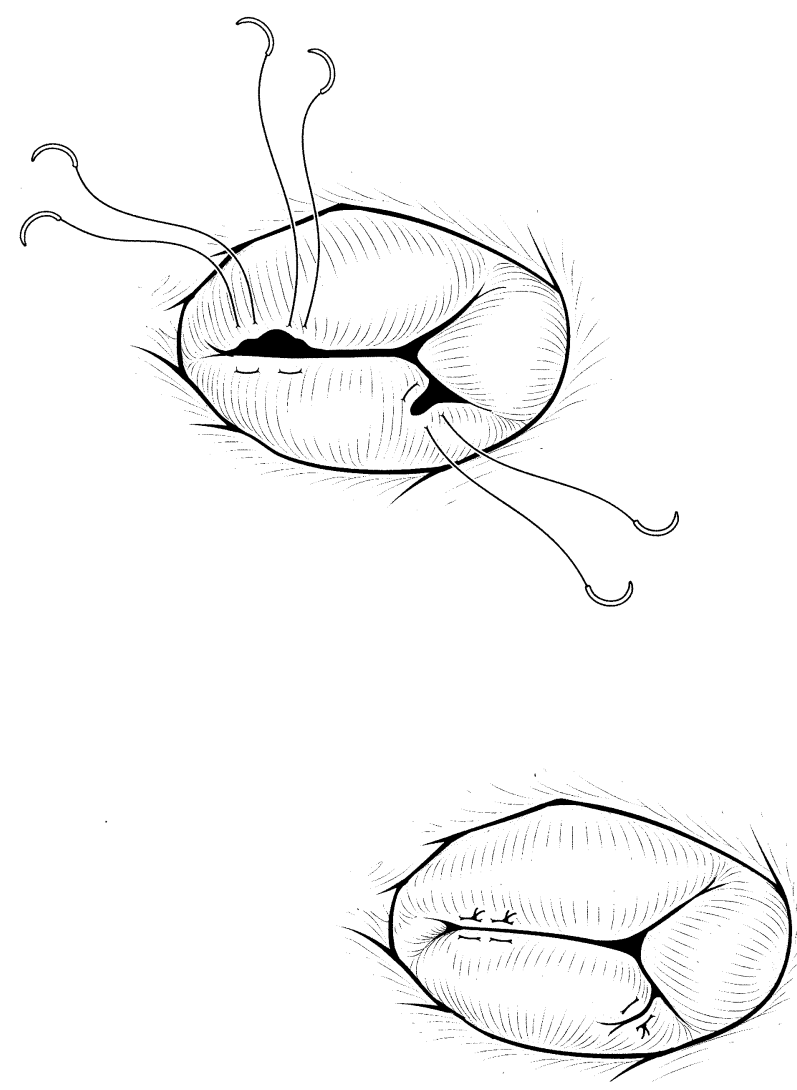

Figure 3. Partial commissure closure and leaflet cleft repair.

0 to $2+$ TR at last follow-up, or an adverse outcome, as defined by 3 to $4+$ TR. Seventeen $(63 \%)$ patients were found to have had a successful outcome at a median follow-up of 26 months (range, 1-82 months). Ten (37\%) patients had a poor outcome at a median follow-up of 13 months (range, 1-75 months). Median age at repair, weight at repair, stage of palliation, tricuspid valve pathology, and length of follow-up were found not to be significantly different between the 2 groups (Table 1). Patients in the unsuccessful outcome group tended to require repair earlier in their palliation. Sixty-five percent (11/17) of patients in the success group achieved at least the hemi-Fontan procedure before requiring tricuspid valve repair compared with only $30 \%(3 / 10)$ in the failure group $(P=.12)$. Concomitant tricuspid valve repair and either the hemi-Fontan procedure or the Fontan procedure were common in both groups (Table 2). In the group achieving a successful late result, 6 of 9 patients who were at the hemi-Fontan procedure stage at the time of their tricuspid valve repair were able to undergo a successful concomitant Fontan procedure.

\section{Repair Technique}

Partial annuloplasty, used in 17 patients, was the most frequently used repair technique. Partial commissure clo- 
TABLE 1. Nonsignificant variables

\begin{tabular}{llcc}
\hline & \multicolumn{2}{c}{ Late outcome } & \\
\cline { 2 - 3 } Variable & $\mathbf{0 - 2 +}$ TR & $\mathbf{3 - 4 +}$ TR & P value \\
\hline Median age, mo (range) & $20(5-56)$ & $4(4-127)$ & .32 \\
Median weight, kg (range) & $8.7(4.5-18.7)$ & $6.1(4.9-35.6)$ & .24 \\
$\begin{array}{l}\text { Median follow-up, mo } \\
\quad \text { (range) }\end{array}$ & $26(1-82)$ & $13(1-75)$ & .08 \\
$\begin{array}{l}\text { Predominant valve pathology } \\
\quad \text { (leaflet prolapse-tethering) }\end{array}$ & $15 / 2$ & $7 / 3$ & .33 \\
\hline
\end{tabular}

Comparison of variables between patients achieving a successful late result and those with a poor late result.

$T R$, Tricuspid valve regurgitation.

TABLE 2. Concomitant procedures performed with tricuspid valve repair

\begin{tabular}{lcc}
\hline & \multicolumn{2}{c}{ Late outcome } \\
\cline { 2 - 3 } Concomitant procedure & $\mathbf{0 - 2 +}$ TR & 3-4+ TR \\
\hline Hemi-Fontan & $6 / 17(35 \%)$ & $7 / 10(70 \%)$ \\
Fontan & $6 / 17(35 \%)$ & $1 / 10(10 \%)$
\end{tabular}

$T R$, Tricuspid valve regurgitation.

TABLE 3. Primary valve pathology as assessed by means of echocardiography ( $P=$ not significant)

\begin{tabular}{lrr}
\hline & \multicolumn{2}{c}{ Late outcome } \\
\cline { 2 - 3 } Valve pathology & $\mathbf{0 - 2 +}$ TR & $\mathbf{3 - 4 +}$ TR \\
\hline Leaflet prolapse & $15 / 17(88 \%)$ & $7 / 10(70 \%)$ \\
Leaflet tethering & $2 / 17(12 \%)$ & $3 / 10(30 \%)$
\end{tabular}

$T R$, Tricuspid valve regurgitation.

sure for localized areas of leaflet prolapse was used in 6 patients. Ring annuloplasty was performed in 5 patients with a median ring size of $28 \mathrm{~mm}$ (range, 25-31 mm). Chordal shortening was used in 3 patients, and papillary muscle advancement and mobilization of the septal leaflet were used in 1 patient each. The repair technique of partial annuloplasty alone, when compared with any other method or combination of methods, significantly predicted 0 to $2+$ TR at late follow-up $(P=.04$, Figure 4$)$.

\section{Tricuspid Valve Regurgitation}

All patients had 3 to $4+$ TR before the operation. There was no difference between the tricuspid valve pathology primarily responsible for the regurgitation, as assessed by means of 2-dimensional and Doppler echocardiography (Table 3). Similar degrees of tricuspid valve annulus dilatation were seen in both groups preoperatively, early postoperatively, and at late follow-up. However, in both groups the median $z$ value decreased after the valve repair. The $z$ value then tended to stay stable in the late success group, whereas it

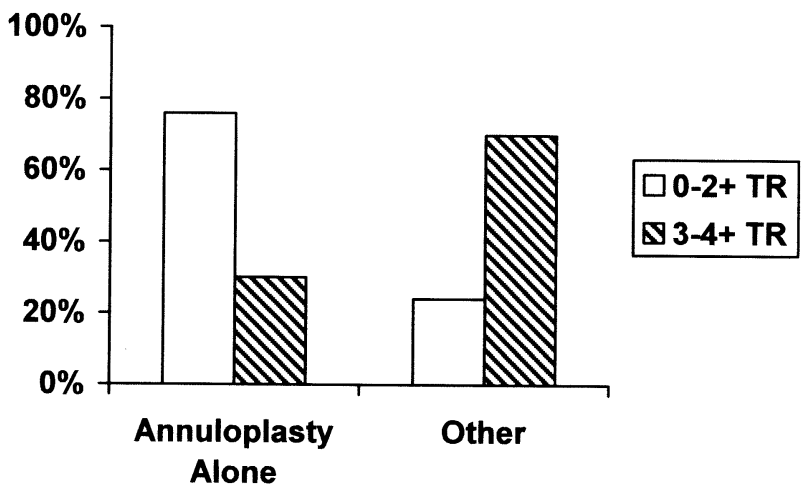

Figure 4. Repair techniques: comparison of patients with a successful outcome and an adverse outcome, revealing a significant difference between partial annuloplasty alone and the need for any other repair technique or combination of techniques $(P=$ .04).

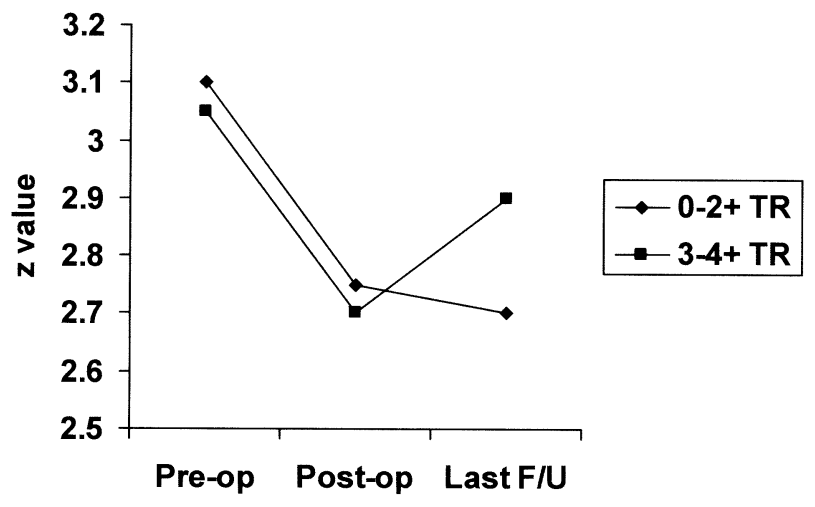

Figure 5. Tricuspid valve $z$ values: comparison of preoperative, early postoperative, and late tricuspid valve $z$ values between the late success and failure groups.

increased slightly in the group with late failure (Figure 5). After tricuspid valve repair, 21 patients had an early successful result as defined by 0 to $2+$ TR in the immediate postoperative period. Of these 21 patients, 15 maintained a durable repair at late follow-up, and 6 had late failure. Six patients had an early unsuccessful repair, 4 of whom continued to have a poor result at late follow-up. The remaining 2 patients with an unsuccessful repair in the early postoperative period underwent rerepair with a successful late outcome (Figure 6). Early successful repair was predictive of late success $(P=.012)$, with a sensitivity of $76 \%$, a specificity of $70 \%$, a positive predictive value of $81 \%$, and a negative predictive value of $64 \%$.

\section{RV Function}

All patients had similar RV function preoperatively, with a median functional grading of normal, ranging from normal to mildly decreased. There was no significant difference 


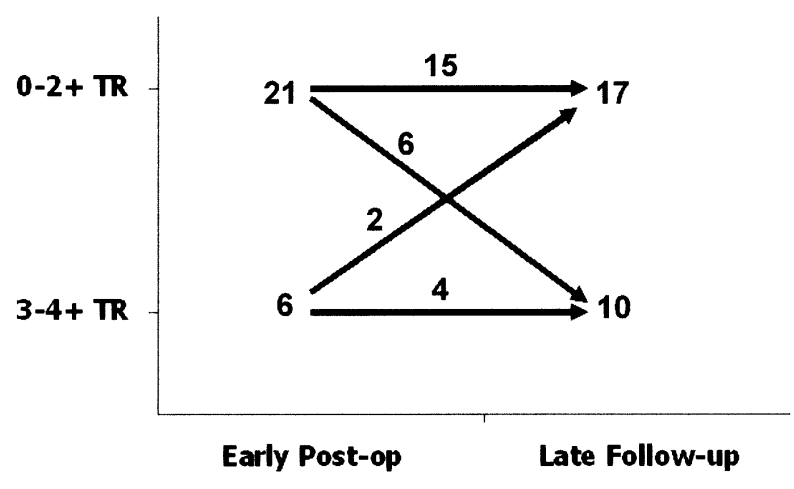

Figure 6. Diagram of the early and late TR after repair. The number of patients in each group and the number of patients crossing over between groups are indicated.

between the late success group and the late failure group with respect to the early postoperative or late follow-up RV function. Although differences failed to reach statistical significance, interesting trends were noted when comparing the groups. These trends were particularly apparent when comparing the 4 groups with early postoperative success and late follow-up success, early success and late failure, early failure and late success (because of successful rerepair), and early failure and continued late failure, as outlined in Figure 6. Those patients with early success and continued late success of repair (Figure 7, A) had preserved RV function both early postoperatively and at late follow-up. Similarly, those patients with an early failure who were salvaged with a rerepair to a successful late outcome (Figure $7, B$ ) had preserved RV function throughout. Conversely, patients with an early success but late failure (Figure 7, C) had poor RV function, even in the early postoperative period, which was persistent at late follow-up. The last group, those patients with both early and late failure (Figure $7, D$ ), initially had preserved RV function in the early postoperative period, which deteriorated at late follow-up.

\section{Patient Outcomes and Survivals}

Of the 17 patients with a successful repair at late follow-up, 15 had achieved their Fontan repair (Figure 8, A). Among this group, there were 14 long-term survivors and 1 death. The death was a witnessed sudden death in a patient with 1+ TR and mild-to-moderate RV dysfunction 2 years after his Fontan operation. Two patients were at a hemi-Fontan stage, one of whom is awaiting his Fontan operation and is considered to be a good Fontan candidate. The other patient underwent a cardiac transplantation for diastolic dysfunction with normal RV systolic function and minimal TR.

There were 10 patients with an unsuccessful repair at late follow-up (Figure 8, B). There were 8 deaths in this group. Two deaths were early operative mortalities, one caused by an intraoperative coronary artery injury and the other by

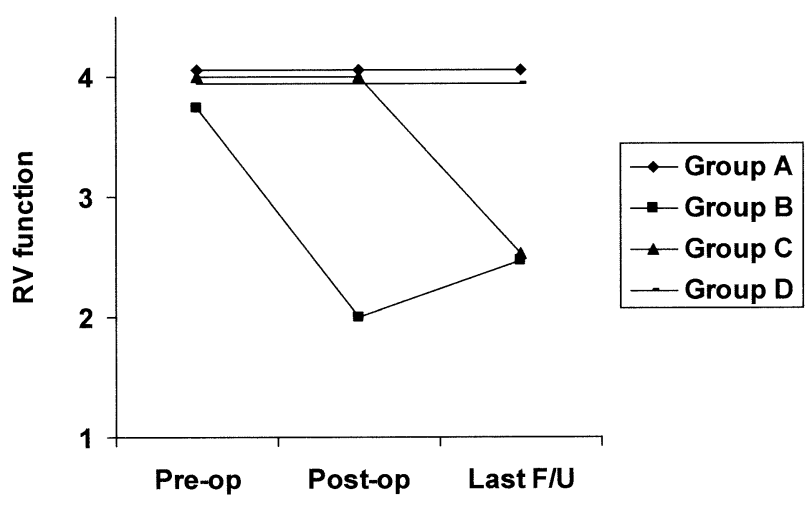

Figure 7. RV function. Scale: 4, normal; 3, mildly decreased; 2, moderately decreased; 1 , severely decreased. Groups: $A$, early success/late success; $B$, early failure/late success; $C$, early failure/late failure; $D$, early success/late failure.

A.

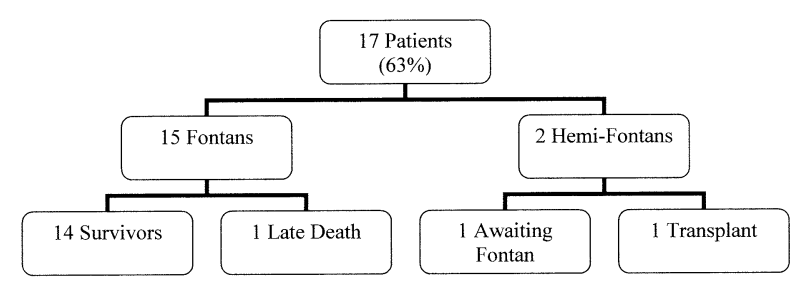

B.

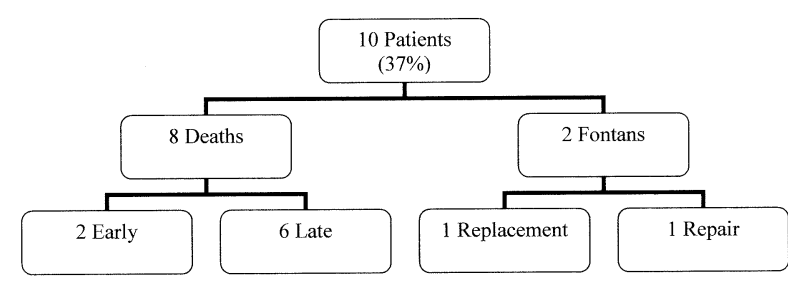

Figure 8. Outcomes of the patients with successful late repair (n $=17, A)$ or unsuccessful late repair $(n=10, B)$.

postoperative RV failure. There were 6 late deaths, all of which had required an attempted rerepair or mechanical valve replacement. Two of the 10 patients in this group are late survivors, both of whom have achieved a successful Fontan procedure. One patient has a mechanical prosthetic valve. The other patient has had a single repair with residual 3+ TR, has normal RV function, and remains in New York Heart Association (NYHA) class I.

Overall survival for the entire cohort was 67\% (18/27). For patients with a successful late repair, survival was $94 \%$ (16/17). In contrast, patients with 3 to $4+$ TR at follow-up had a survival of $20 \%(2 / 10)$, a difference that was statistically significant $(P=.0002)$. With respect to RV function, 
comparison of the survivals of those patients with normal to mildly decreased function (86\% [18/21]) and those with moderate-to-severe dysfunction $(0 \%$ [0/6]) similarly revealed a significant difference in survival $(P=.0003)$. Of the 18 late survivors, 17 are in NYHA class I, and 1 is in NYHA class II.

\section{Study Limitations}

The limitations of this study are primarily related to the retrospective nature of the analysis and to the limited number of patients. In addition, the complex and multifactorial nature of TR in HLHS makes defining the specific variables responsible for the outcomes of repair and other late outcomes difficult.

There is also a risk of selection bias because there might have been patients with severe TR who were not captured. Although cardiac transplantation for severe TR and severe $\mathrm{RV}$ was accounted for, it is possible that other patients might not be included in this cohort. These patients might have had significant TR and RV dysfunction and died at home or had other surgical intervention or died at outside institutions.

\section{Discussion}

\section{Tricuspid Valve Repair}

Overall, these data demonstrate that tricuspid valve repair can be successfully accomplished in the majority of patients with HLHS, even in the face of severe TR. Repair can be performed as a sole procedure or in conjunction with a hemi-Fontan or Fontan procedure. Successful repair in the early postoperative period is significantly predictive of continued excellent valve function and preserved RV performance at late follow-up. Partial annuloplasty was found to be significantly associated with a successful late outcome. However, partial annuloplasty might be a surrogate for other factors, which were not detected. These factors might include variables, such as fine differences in tricuspid valve anatomy not delineated by echocardiography, subclinical RV dysfunction, or subtle coronary artery insufficiency. Several different repair techniques were successfully used, and the method of repair should be individualized to the particular anatomy encountered. Similarly, the trend toward earlier operation in the group with an unsuccessful late outcome likely represents a surrogate for a worse subgroup of TR that tends to present earlier, rather than an indication that repair should be delayed at all costs.

\section{Interaction of TR and RV Function}

TR, particularly in the setting of HLHS, is the result of several complex and interrelated variables. RV function appears to be one of the variables that has an important role in the natural history of tricuspid regurgitation. When comparing patients with a successful early postoperative result that either continued to have 0 to $2+$ TR or went on to late failure, those patients who maintained a successful repair had significantly better RV function.

However, the contribution of any given factor, such as $\mathrm{RV}$ function, to the development of TR is likely variable from patient to patient. This variable influence of RV function on TR might be illustrated by trends that were demonstrated in comparing the early postoperative results with the late outcomes of the various groups. Patients with both an early postoperative and late successful outcome had preserved RV function throughout the period of follow-up. Similarly, patients with an initial unsuccessful repair who achieved a successful late result with rerepair also had preserved RV function, both demonstrating the importance of RV function in tricuspid valve competence. In contrast, patients with an early successful repair followed by late failure had more decreased RV function in both early and late follow-up, suggesting that RV dysfunction might have played an important role in the late deterioration of tricuspid valve function. Conversely, in patients with poor tricuspid valve competence both immediately after repair and at late follow-up, RV function was initially preserved and later decreased, implying an adverse effect of TR on RV function.

\section{Survival and Outcomes}

Despite remarkable improvements in survivals for HLHS over the past 2 decades, interstage and 5-year mortality remains significant. ${ }^{1-5}$ An important factor in this ongoing attrition is the development of significant TR, which has been reported to occur in from $8.5 \%$ to $16 \%$ of patients with HLHS. $^{7,8}$ In a previous publication from our institution, ${ }^{9}$ $14 \%$ of patients had $2+$ TR or greater after a Norwood procedure for HLHS. TR has long been recognized as a risk factor for Fontan procedure survival and has even been suggested as a relative contraindication to pursuing stage palliation for HLHS. ${ }^{7,8,10}$ However, tricuspid valve repair can be successfully performed and contributes to improved outcomes. Overall, the percentages of patients progressing to a successful Fontan procedure and the overall survivals after tricuspid valve repair are excellent in this very highrisk subpopulation of patients with HLHS.

Patients with an initially successful repair but poor RV function tended to do poorly as a group because deteriorating tricuspid valve function often developed. Patients with an initially poor result for repair but preserved RV function can achieve a good result with a rerepair. However, when $\mathrm{RV}$ dysfunction develops over time in those patients with residual TR, the prognosis is poor. The poor outcome associated with the latter subgroup suggests that these patients might benefit from early consideration for cardiac transplantation. 


\section{References}

1. Tweddell JS, Hoffman GM, Mussatto KA, Fedderly RT, Berger S, Jaquiss RD, et al. Improved survival of patients undergoing palliation of hypoplastic left heart syndrome: lessons learned from 115 consecutive patients. Circulation. 2002;106(suppl I):I82-9.

2. Azakie A, Merklinger SL, McCrindle BW, Van Arsdell GS, Lee KJ, Benson LN, et al. Evolving strategies and improving outcomes of the modified Norwood procedure: a 10-year single-institution experience. Ann Thorac Surg. 2001;72:1348-53.

3. Ishino K, Stumper O, De Giovanni J, Silove E, Wright J, Sethia B, et al. The modified Norwood procedure for hypoplastic left heart syndrome: early to intermediate results of 120 patients with particular reference to aortic arch repair. J Thorac Cardiovasc Surg. 1999;117: 920-30.

4. Mahle WT, Spray TL, Gaynor JW, Clark BJ III. Unexpected death after reconstructive surgery for hypoplastic left heart syndrome. Ann Thorac Surg. 2001;71:61-5.

5. Lloyd TR. Prognosis of the hypoplastic left heat syndrome. Prog Pediatr Cardiol. 1996;5:57-64.

6. Daubeney PE, Blackstone EH, Weintraub RG, Slavik Z, Scanlon J, Webber S. Relationship of the dimension of cardiac structures to body size: an echocardiographic study in normal infants and children. Cardiol Young. 1999;9(4):402-10.

7. Chang AC, Farrell PE Jr, Murdison KA, Baffa JM, Barber G, Norwood WI, et al. Hypoplastic left heart syndrome: hemodynamic and angiographic assessment after initial reconstructive surgery and relevance of modified Fontan procedure. J Am Coll Cardiol. 1991;17(5): 1143-9.

8. Barber G, Helton JG, Aglira BA, Chin AJ, Murphy JD, Pigott JD, et al. The significance of tricuspid regurgitation in hypoplastic left heart syndrome. Am Heart J. 1988;116:1563-7.

9. Reyes A II, Bove EL, Mosca RS, Kulik TJ, Ludomirsky A. Tricuspid valve repair in children with hypoplastic left heart syndrome during staged surgical reconstruction. Circulation. 1997;96(suppl II):II341-3.

10. Ghoussat A, Fontan F, Besse P, Vallot F, Chauve A, Bricaud H. Selection criteria for Fontan's operation. In: Anderson RH, Shinebourne EA, editors. Pediatric cardiology. Edinburgh: Churchill Livingstone; 1977. p. 559-66.

\section{Discussion}

Dr Thomas L. Spray (Philadelphia, Pa). I am pleased to have the opportunity to discuss this excellent and timely contribution from Dr Ohye and his colleagues at the University of Michigan. Obviously, as he mentioned, as success rates for staged reconstruction for HLHS continue to improve, the problems that affect long-term outcome, such as TR, are gaining increasing importance. The authors have made an excellent attempt to study the effects of valve repair for TR after the Norwood operation for HLHS, with results that are good considering the fact that the patient population is quite diverse. As in most excellent and timely series, however, there are many questions stimulated by their discussion that I think will require ongoing investigation. I have several questions for the authors.

First, the authors described a series of 28 patients who had significant TR and underwent valve repair, but in other studies from their own institution, they reported an incidence of $14 \%$ of significant valve regurgitation after the Norwood operation. That would suggest that there are a large number of patients not represented in this series who have significant TR and yet did not undergo valve repair. I wonder if you could comment on the clinical criteria that you used to determine the timing of tricuspid repair and which patients really seemed to be appropriate for the procedure?

Was severe TR associated with stage I reconstruction or with recurrent arch obstruction, for example, in your series? Were there other clinical criteria that forced you to intervene with tricuspid repair? This is especially important because, as you noted, one of your patients who underwent a failed repair actually did well in follow-up, with good ventricular function and a Fontan procedure. Therefore why do you repair these valves, and how important is it to repair them?

Second, our own experience would suggest that moderate or greater TR that is present at birth or immediately after the firststage reconstruction presages a very poor long-term outcome. I wonder if you have had a similar experience and if you could comment on that.

And finally, our own experience suggests that you get improvement in atrioventricular valve function at the hemi-Fontan procedure just from volume unloading of the ventricle, and I noted in your article and in your series that several patients underwent valvuloplasty at the time of the hemi-Fontan or second-stage operation. Do you think that valve repair should be performed in all patients with significant TR at the second operation, or would you allow some patients to volume unload and see whether their TR improves?

Dr Ohye. Thank you for your comments, Dr Spray. To address your first comment about the $14 \%$ presented in a previous article from our institution, those patients had greater than or equal to $2+$ TR, and we limited our patients only to those with 3 to $4+$ TR. The range in the literature for "significant" TR ranges from about $8.5 \%$ to $16 \%$, and therefore these patients should represent a good capture of the cases of significant, or 3 to $4+$, TR.

As to the question regarding the time of presentation of TR and the indications and timing of repair, because these patients had 3 to $4+\mathrm{TR}$, the repair was mandated by the presence and degree of TR. None of these patients had 2+ TR or less and would have been considered for expectant management with hopes of improvement with the reduction in volume overload associated with additional staged palliation. Of the patients who were at their Norwood stage, which were 15 patients, all underwent a concomitant hemi-Fontan procedure. At the time of the detection of their TR (before hemiFontan work-up), they would require some procedure to improve their pulmonary blood flow. The options would be either to reshunt them and do a repair or do a hemi-Fontan procedure and repair. We have selected to perform a concomitant hemi-Fontan procedure because, as you pointed out, volume offloading the ventricle at the hemi-Fontan procedure might, in fact, help. Of the patients who were at their hemi-Fontan stage, which included 10 patients, 7 underwent a successful concomitant Fontan procedure.

Thus in terms of timing, repair was mandated in this population by the fact that they had significant TR. Because of the fact that we only selected those patients with 3 to $4+$ TR, I believe there is little argument that they would not have done well without repair. We did not limit progression to further palliative stages in patients who were otherwise good anatomic and hemodynamic candidates on the basis of the presence of TR.

We have also found that the patients who present at birth with 3 to $4+$ TR are a very difficult group of patients. Those patients with 3 to $4+$ TR are also considered for transplantation at that stage rather than a Norwood operation. However, this study only addressed patients with 3 to $4+$ TR after a successful Norwood operation. 
In regard to volume unloading at the second stage, again, I would reiterate that these patients had 3 to $4+\mathrm{TR}$, and we would not be comfortable simply doing a hemi-Fontan procedure with expectant management. I believe that TR does get better somewhat with volume overloading, but that is really not sufficient to address TR of the significance present in these patients.

Dr Jan M. Quaegebeur (New York, NY). I have a question about the timing of the tricuspid valve repair. In your abstract the mean age is 24 months. In our experience, when patients have tricuspid valve incompetence, it is present very early in their disease process, and we would tend to perform these repairs at the age of 6 months. So why do you think that there is such a scatter from young patients to quite older patients when you did these repairs, and what are your present recommendations?

Dr Ohye. Our recommendations are that the valves need to be repaired when the TR is significant, regardless of age of stage of palliation. I believe our patients presented throughout their course of staged repair because of the multifactorial nature of the disease. Some patients have intrinsic valve abnormalities and might present earlier, as Dr Spray said, even at the time of birth. Some of these patients might have TR as a result of their ventricular dysfunction and then tend to present later.

\section{Targeted}

The Journal of Thoracic and Cardiovascular Surgery gives you two tables of contents.

The condensed table of contents tells you at a glance what topics and authors are presented each month. The expanded table of contents gives you a brief abstract of each article. You select only those articles of most interest to you for further reading. 